\title{
Neutron monitor time-delay measurements to track cosmic ray spectral variation due to solar modulation at high and low cutoff rigidity
}

\author{
C. Banglieng ${ }^{* 1,2}$, H. Janthaloet ${ }^{1}$, D. Ruffolo ${ }^{1}$, A. Sáiz ${ }^{1}$, W. Mitthumsiri ${ }^{1}$, P. Evenson ${ }^{3}$, \\ T. Nutaro ${ }^{4}$, R. Pyle ${ }^{5}$, S. Seunarine ${ }^{6}$, and J. Madsen ${ }^{6}$, \\ ${ }^{1}$ Department of Physics, Faculty of Science, Mahidol University, Bangkok 10400, Thailand. \\ ${ }^{2}$ National Astronomical Research Institute of Thailand (NARIT), Chiang Mai 50180, Thailand. \\ ${ }^{3}$ Bartol Research Institute and Department of Physics and Astronomy, University of Delaware, \\ Newark, DE 19716, USA. \\ ${ }^{4}$ Department of Physics, Faculty of Science, Ubon Ratchathani University, Ubon Ratchathani \\ 34190, Thailand. \\ ${ }^{5}$ Pyle Consulting Group, Inc., St. Charles, IL 60174, USA. \\ ${ }^{6}$ Department of Physics, University of Wisconsin, River Falls, WI 54022, USA. \\ E-mail: bchanoknanegmail.com, hannarong.janestudent.mahidol.ac.th, \\ david.ruf@mahidol.ac.th, alejandro.sai@mahidol.ac.th, \\ warit.mit@mahidol.ac.th, evenson@udel.edu, tnutaro@yahoo.com, \\ rogerpyle@gmail.com, surujhdeo.seunarineduwrf.edu, \\ james.madsen@uwrf.edu
}

\begin{abstract}
The solar modulation of Galactic cosmic rays (GCRs) provides remote sensing of heliospheric conditions throughout the $\sim 11$-yr sunspot cycle and $\sim 22$-yr solar magnetic cycle. Neutron monitors (NMs) can measure cosmic ray rates above a cutoff rigidity with high precision $(\sim 0.1 \%)$ over such time scales. To avoid systematic uncertainties in comparing NM count rates from different stations, here we study solar modulation of the GCR spectrum by independently using neutron time-delay histograms to determine the leader fraction $L$, i.e., the inverse neutron multiplicity, as a proxy of the GCR spectral index above the cutoff. Data were collected using specialized electronics at two high-altitude NM stations: 1) the Princess Sirindhorn Neutron Monitor (PSNM) at Doi Inthanon, Thailand, with the world's highest vertical geomagnetic cutoff rigidity, $16.8 \mathrm{GV}$, from 2007 December to 2018 September, and 2) the South Pole Neutron Monitor with an atmospherelimited cutoff of $\sim 1 \mathrm{GV}$, from 2013 December to 2018 September. After correcting for pressure and (in Thailand) precipitable water vapor, we find that $L$ is roughly correlated with the count rate, but also exhibits hysteresis that indicates a change in spectral shape after the change in solar magnetic polarity. These methods enhance the high-precision GCR spectral information from the worldwide NM network and extend it to higher rigidity.
\end{abstract}

36th International Cosmic Ray Conference -ICRC2019-

July 24th - August 1st, 2019

Madison, WI, U.S.A.

${ }^{*}$ Speaker. 


\section{Introduction}

Neutron monitors (NMs) are ground-based detectors of secondary neutrons from nuclear cascades in the Earth's atmosphere. NMs have high precisely measured the variation of Galactic cosmic rays due to the solar modulation, i.e., variations with the roughly 11-year sunspot cycle and 22-year solar magnetic cycle. The magnetic latitude on the Earth's surface can be used to provide a cutoff rigidity, i.e., the minimum momentum per charge for cosmic rays to arrive at the atmosphere above the monitor. The effective geomagnetic cutoff for vertically arriving particles varies from $\sim 17 \mathrm{GV}$ in and near Thailand to $<0.01 \mathrm{GV}$ in some polar regions. Therefore, the worldwide NM network can provide some information on GCR spectral variations in an intermediate rigidity range when comparing count rates at different cutoff rigidities. Although individual NMs can measure the cosmic ray flux with high statistical precision, the comparison of long-term data from different stations involves many effects such as different NM configurations, electronic dead time, structures surrounding the stations, seasonal effects, and atmospheric structure as well as long-term changes in the geomagnetic field.

Therefore, we have developed a capability to precisely track spectral variations using data from an individual NM station. We developed the specialized electronics to record neutron time-delay histograms[1] and techniques to remove the effect of chance coincidences and extract the "leader fraction" $L$ (inverse multiplicity) [2]. Analyzing $L$ from a ship-borne latitude survey confirmed that $L$ is sensitive to the primary cosmic ray spectrum[3]. The Princess Sirindhorn Neutron Monitor (PSNM) at Doi Inthanon, Thailand, which has the world's highest vertical cutoff rigidity $(\approx 16.7$ GV), is the first fixed station using these electronics. In addition, the South Pole Neutron Monitor at Antarctica, which has a low atmosphere-limited cutoff rigidity, started using these electronics in 2013 December. Here we present a long-term dataset of the leader fraction at Doi Inthanon from 2007 December to 2018 April, which spans a full cycle of solar modulation, including the all-time cosmic ray maximum of 2009 and minimum near the end of 2014, and also at South Pole from 2013 December to 2018 April, which started near the cosmic-ray minimum.

\section{Neutron monitor time-delay observations}

PSNM is located at the summit of Doi Inthanon, Thailand's highest mountain, at latitude $18.59^{\circ} \mathrm{N}$, longitude $98.49^{\circ} \mathrm{E}$ and altitude $2560 \mathrm{~m}$, with a standard 18 -counter NM64 configuration. The South Pole NM at an altitude of $2828 \mathrm{~m}$ has three 1NM64 detectors.

Tables 1 and 2 describe the development of the firmware in the electronics and the software in the data acquisition system used to collect time delay histograms at PSNM and the South Pole NM. The normalization periods are characterized depending on significant changes in firmware and/or software. Note that the changes do not affect the separate circuitry used to record count rates. The original 600 series firmware used to collect time-delay histograms at Doi Inthanon and South Pole during the earlier periods DI- 1 and SP-1 was limited to collect only at $16 \mathrm{~Hz}$ per tube resulting in a "first-pulse bias" described by [2]. Then starting on 2011 January 15, we upgraded to the 700 series that can collect time delays at up to $48 \mathrm{~Hz}$ per tube allowing data collection at the full rate at Doi Inthanon $(\sim 34 \mathrm{~Hz})$ for improved statistics. 
Table 1: Time periods of neutron time-delay data collection from the 18NM64 at Doi Inthanon, Thailand

\begin{tabular}{lllccc}
\hline $\begin{array}{l}\text { Normalization } \\
\text { period }\end{array}$ & Start date & End date & Cadence & $\begin{array}{c}\text { Firmware series } \\
\text { (Number of tubes) }\end{array}$ & Software version $^{a}$ \\
\hline \hline DI-1a & 2007 Dec 9 & 2009 Jun 28 & Daily & $600(18)$ & - \\
DI-1b & 2009 Jun 29 & 2011 Jan 15 & Hourly & $600(18)$ & - \\
DI-2 & 2011 Jan 15 & 2014 Feb 8 & Hourly & $700(18)$ & - \\
DI-3 & 2014 Feb 8 & 2014 Jun 11 & Hourly & $700(17)$ & - \\
DI-4a & 2014 Jun 11 & 2014 Dec 6 & Daily & $800(18)$ & $8.34-8.46$ \\
DI-4b & 2014 Dec 7 & 2015 Mar 3 & Hourly & $800(18)$ & 8.47 \\
DI-5 & 2015 Mar 3 & 2015 May 29 & Hourly & $800(18)$ & 8.50 \\
DI-6a & 2015 May 31 & 2016 May 17 & Hourly & $600(6), 800(12)$ & $8.50-8.82$ \\
DI-6b & 2016 May 18 & 2016 Jun 30 & Hourly & $600(6), 800(12)$ & 8.82 \\
DI-7 & 2016 Jun 30 & 2017 Jun 12 & Hourly & $800(18)$ & 8.82 \\
DI-8 & 2017 Jun 12 & 2017 Aug 3 & Hourly & 600(6), 700(6), 800(6) & $8.91-8.93$ \\
DI-9 & 2017 Aug 4 & 2018 Apr 19 & Hourly & $800(18)$ & $8.93-8.124$ \\
\hline
\end{tabular}

${ }^{a}$ Only relevant for 800 firmware.

On 2014 February 8, we started the process of upgrading to the 800-series firmware on Tube 1 but this initial version of firmware did not provide usable histograms so during DI-3 we analyze only 700-series firmware on the remaining 17 tubes. The complete development of the 800-series firmware was used for 18 counter tubes starting on 2014 June 11 in order to calculate absolute times and cross-tube time delays, which are not used in the present analysis [4]. Because the absolute time delays are calculated by the data acquisition software, they can depend on changes in the software version or computer. Starting with the 800 series during DI-4a, there were problems of software that can be corrected with a daily cadence, and after that hourly histograms were usable again. On 2014 December 7, we swapped counter tubes number 1 and 18 in order to study the effect of the tube position. During DI-5, we changed the software version to record time delays and the normalization of $L$ was lower than during DI-4. We did not observe any differences in $L$ associated with further changes in the software version. At the same time, the South Pole NM used the 800 series for the complete set of 3 counter tubes, but the time-delay histograms recorded during 2014 Nov 28 - 2015 Mar 2 were not useable. After 2015 Mar 3, the start of SP-2, the South Pole NM used the 800-series firmware to collect time delay histograms from all 3 counter tubes.

To normalize $L$ from the 600-series firmware, we did an experiment to calibrate $L$ of 600 series to the 800 series during DI-6 as detailed in Table 1. We also performed a calibration for South Pole during SP-3 between 2017 Dec 18 and 2018 Jan 30, and after that we again used the 800 series for all tubes (SP-4). On 2016 May 18, we upgraded the data acquisition computer for PSNM and we found $L$ from the newer computer to be slightly higher than $L$ from the older computer. We performed a calibration again during DI-8 as detailed in Table 1. Afterward, PSNM has used the 800-series to collect time-delay histograms. Our analysis considers data up to 2018 April 19. 
Table 2: Time periods of neutron time-delay data collection from the $3 \times 1$ NM64 at South Pole, Antarctica

\begin{tabular}{llllcc}
\hline $\begin{array}{l}\text { Normalization } \\
\text { period }\end{array}$ & Start date & End date & Cadence & $\begin{array}{c}\text { Firmware series } \\
\text { (Number of tubes) }\end{array}$ & Software version $^{a}$ \\
\hline \hline SP-1 & 2013 Dec 9 & 2014 Nov 27 & Hourly & $600(1)$ & - \\
SP-2a & 2015 Mar 3 & 2015 Mar 5 & Hourly & $800(3)$ & 8.47 \\
SP-2b & 2015 Mar 6 & 2017 Dec 9 & Hourly & $800(3)$ & $8.50-8.80$ \\
SP-3 & 2017 Dec 18 & 2018 Jan 30 & Hourly & 600 (1), 800 (2) & 8.80 \\
SP-4 & 2018 Jan 31 & 2018 Apr 19 & Hourly & $800(3)$ & 8.80 \\
\hline
\end{tabular}

${ }^{a}$ Only relevant for 800 firmware.

\section{Leader fraction analysis}

\subsection{Extraction of the leader fraction}

The leader fraction $L$ is the fraction of neutron counts that did not follow another count in the same counter tube due to the same primary cosmic ray. Ref. [2] developed methods to statistically remove the effect of chance coincidences to extract the leader fraction. More energetic primary cosmic rays lead to more energetic secondary particles that produce more neutrons when interacting inside the neutron monitor (typically in the lead producer), which can then be detected as sequences of more counts in the same tube with shorter time delays. Thus a harder cosmic ray spectrum with a lower spectral index leads to a lower leader fraction. Three analysis methods were described by [2] and here the first is used to calculate the leader fraction $L$.

\subsection{Correction and Normalization of the Leader Fraction}

NM count rates are always corrected for pressure variation, and the leader fraction $L$ has been found to vary with $P$ as well. Moreover, at the tropical location of Doi Inthanon, water vapor variation affects both $L$ and $C$. Even though the effect of water vapor on the count rate is small, with a peak-to-peak seasonal variation of $0.3 \%$, it is significant for studying solar modulation at Doi Inthanon because the peak-to-peak variation due to solar modulation is 3\% [7]. In this work, we corrected $L$ and $C$ based on Precipitable Water Vapor (PWV), i.e., the height of liquid water (in $\mathrm{mm}$ ) that would result if all the water vapor in the atmosphere were to condense at the surface, at Doi Inthanon as determined from the Global Atmospheric Data Assimilation (GDAS) database (see http://ready.arl.noaa.gov/gdas1.php). The South Pole is an extremely dry location, so the effect of atmospheric water vapor is negligible.

The pressure and water vapor corrected $L$ for each counter tube is different, mainly due to its position and electronic dead time. We first normalized $L$ from each tube to a standard set of tubes based on statistics of measured $L$ ratios. These ratios were slightly dependent on the firmware, so we used the mean ratios for each firmware. There is a systematic effect that provides a higher $L$ prior to software version 8.50. Therefore, we performed an experiment on 2018 Mar 20 to simultaneously collect time-delay histograms using software version 8.47 and 8.117 . The 
mean ratio of $L$ as determined from these two software versions was used to normalize $L$ from normalization period DI-4. Comparing the tube calibration between 600 and 800 series firmware during DI-6 and DI-8, we find a different ratio of $L$ values. We obtained consistent results when using the mean ratio of $L$ from normalization period DI-8 to normalize $L$ from 600- and 700series firmware to 800 series firmware. After that normalization, $L$ was averaged over all tubes that provided good data. The results are shown in Figure 1.

For South Pole, we also performed a calibration of 600- and 800-series firmware during SP3 , and this was used to normalize $L$ from SP-1. The results are shown in Figure 2. However the solar modulation conditions during these time periods were very different, so we consider the normalization of $L$ from SP-4 to be uncertain, as included by blue color in Figure 2.

\section{Spectral variations of galactic cosmic rays}

To examine GCR spectral variations over the past solar cycle, Figure 3 shows three-month averaged data for the leader fraction $L$ vs. count rate $C$ over the whole time period of analysis from Doi Inthanon, Thailand. The data are averaged over three months to reduce the systematic uncertainty from the water vapor correction and the statistical uncertainty. The number at each point indicates the order of the three-month period, starting with "1" for 2007 Dec - 2008 Feb and ending with "42" for 2018 Mar - Apr (truncated to April when our analysis ends). Figure 4 shows monthly averaged data for $L$ vs. $C$ from South Pole. The South Pole data are shown for each month. Although the total count rate is $\sim 300 \mathrm{~s}^{-1}$ compared with $\approx 620 \mathrm{~s}^{-1}$ for Doi Inthanon, South Pole data do not have a water vapor effect with an associated systematic uncertainty, and the total variation of $L$ over the solar cycle is much greater, i.e., about $6 \times 10^{-3}$ for South Pole compared with $8 \times 10^{-4}$ for Doi Inthanon. For these reasons the monthly $L$ data from South Pole provide useful data for studying solar modulation, currently for half a solar cycle. The numbers in Figure 4 start from "1" for 2013 Dec and end at " 53 " for 2018 Apr. In each case $C$ measures the sum of the GCR response function (i.e., spectrum times NM yield function) for each species, integrated over rigidity above the cutoff, i.e., for rigidity $\gtrsim 16.7 \mathrm{GV}$ at Doi Inthanon and $\gtrsim 1 \mathrm{GV}$ at South Pole. For each station $L$ indicates the GCR spectral index over the rigidity range of the NM response.

A basic concept of solar modulation is that with increasing solar activity, the cosmic ray spectrum "rolls" downward from the local interstellar spectrum, and with decreasing solar activity, the spectrum "unrolls" upward again. The change in spectrum is much greater at low rigidity, while at sufficiently high rigidity there is almost no solar modulation. Thus we expect that as $C$ decreases, $L$ will also decrease and vice-versa. If the GCR spectral shape were the same during the rolling and unrolling, e.g., as in the force-field model of solar modulation, we would expect that $L$ vs. $C$ would follow the same path during downward and upward trajectories.

For the South Pole NM, the normalization of the initial data during SP-1 (2013 Dec to 2014 Nov) is uncertain, as indicated by blue coloring in Figure 4. After a three-month data gap, the data from 2015 March (point "16") to 2018 April ("53") follow a consistent, nearly linear trend. This is consistent with "unrolling" of the GCR spectrum in the 1-10 GV range that dominated the variation in South Pole data with solar modulation as solar activity weakened.

For PSNM at Doi Inthanon, recording $L$ and $C$ over a full solar cycle, we observe a hysteresis loop, implying a change in the GCR spectral shape above the cutoff rigidity of $\approx 16.7 \mathrm{GV}$. Note that 


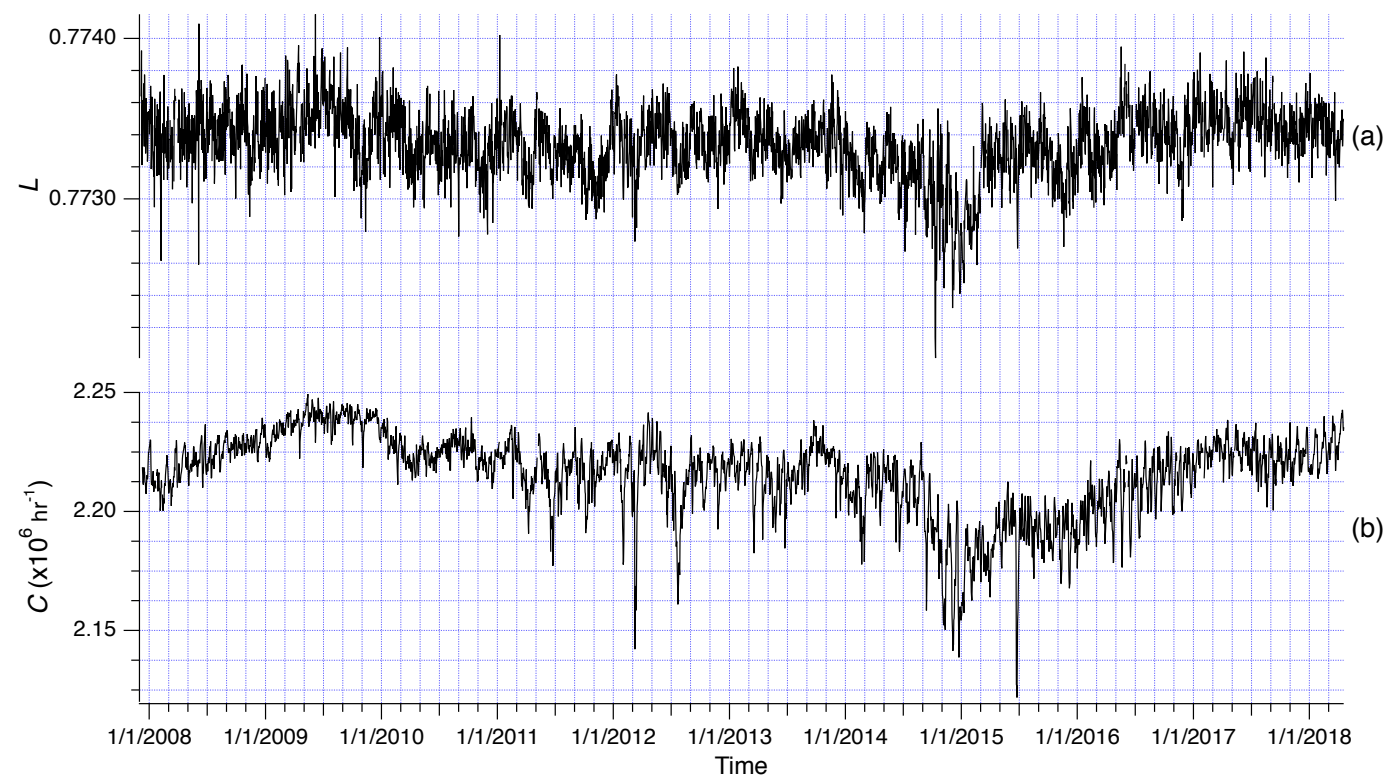

Figure 1: Daily average data of (a) the leader fraction $L$ and (b) count rate $C$ vs. time in the 18NM64 at Doi Inthanon, Thailand at vertical cutoff rigidity $\approx 16.7 \mathrm{GV}$ during 2007 Dec 9 to 2018 Apr 19 .

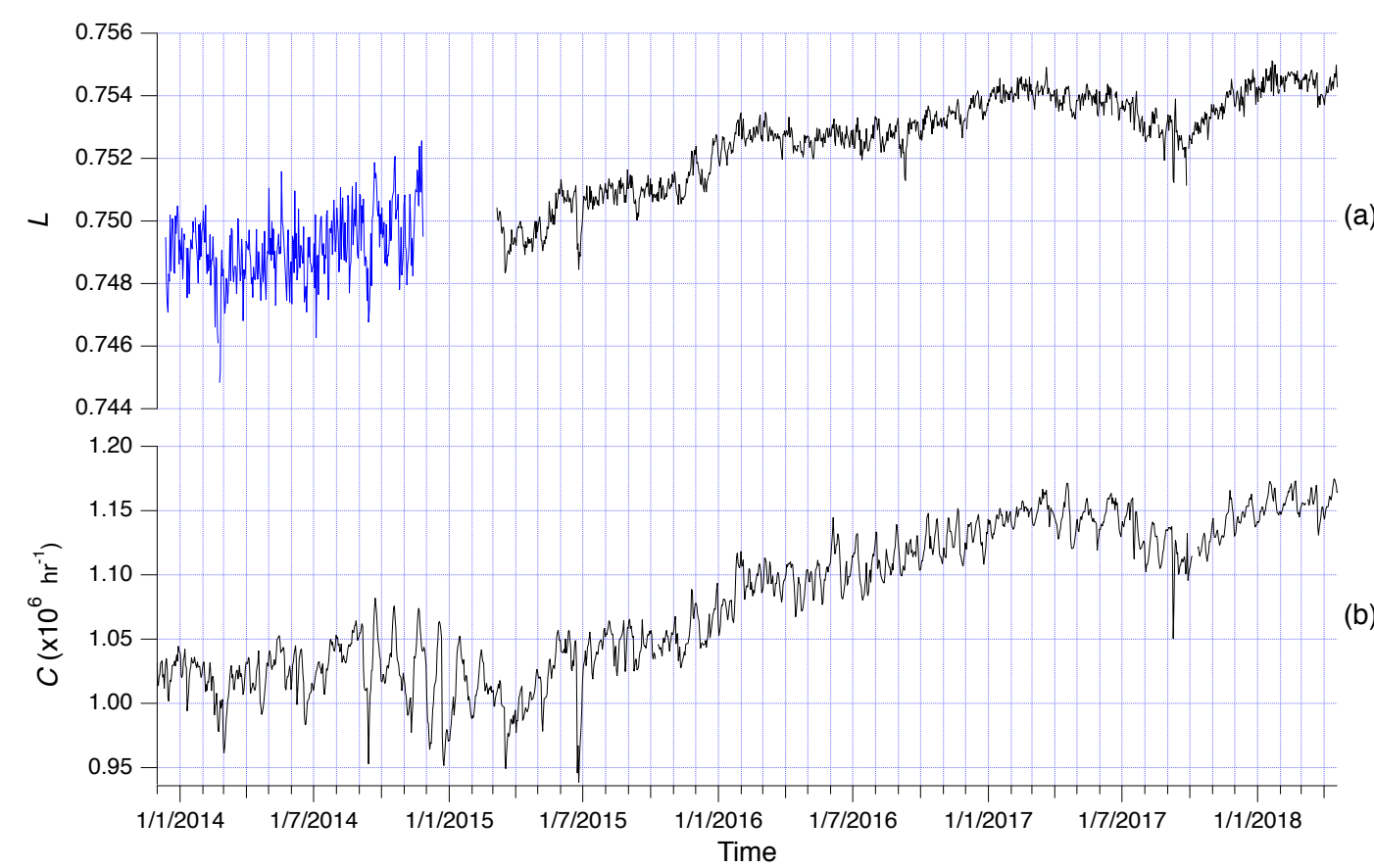

Figure 2: Daily average data of (a) the leader fraction $L$ and (b) count rate $C$ vs. time from the $3 \times 1$ NM64 at South Pole, Antarctica at vertical cutoff rigidity $\lesssim 0.1 \mathrm{GV}$ during 2013 Dec 9 to 2018 Apr 19. Blue coloring indicates that the relative normalization is uncertain for these data. 
the time period of declining cosmic ray rate was associated with solar modulation from the $A<0$ phase of the solar cycle (with mostly inward magnetic field at the northern hemisphere of the Sun) as its effects propagated through the heliosphere, and the rising cosmic ray rate was associated with the $A>0$ phase. Thus our results imply a change in spectral shape with a change in solar magnetic polarity. Such changes have been reported from comparisons with NM data at lower rigidity $[5,6,7]$.

During 2007 Dec to 2014 Nov, $C$ and $L$ at Doi Inthanon declined along a roughly linear trend, which is again consistent with rolling of the spectrum with greater variation at lower rigidity, so that the spectral index decreased along with the count rate. However, shortly after the cosmic ray minimum in early 2015, $L$ shot upward to a nearly maximal value while $C$ increased more gradually. This is qualitatively different from the pattern observed for the South Pole. This implies a significant reorganization of the GCR spectrum above $\approx 16.7 \mathrm{GV}$ within a short time period in early 2015. There was little change in the count rate while the spectral index rapidly increased, indicating a recovery of the GCR flux at about $17 \mathrm{GV}$ while the flux at higher energy had little or no increase or perhaps even declined to result in the nearly constant count rate. In other words, the flux near $17 \mathrm{GV}$ started rising before the flux at higher energy.

Since mid-2015, the count rate at Doi Inthanon mostly had a gradual increase (punctuated by sharp 27-day variations) while the spectral index remained nearly constant, in contrast with the behavior during 2007-2014 in the $A<0$ phase of modulation. During 2007-2014, the changing spectral index implied that the GCR spectrum rolled downward with a significantly stronger decrease near the cutoff rigidity, so that the spectrum became harder as the count rate decreased. However, starting in mid-2015 (in the $A>0$ phase of modulation) the nearly constant spectral index implies a nearly rigidity-independent increase in the flux over a significant rigidity range above the cutoff. Thus the spectral shape and spectral variations above $17 \mathrm{GV}$ were substantially different during the cosmic ray decline (for $A<0$ ) and cosmic ray rise (for $A>0$ ).

\section{Acknowledgments}

This work was partially supported by the Thailand Research Fund award RTA5980003 and the US National Science Foundation awards PLR-1245939 and PLR1341562, and their predecessors. We thank Pradiphat Muangha, Ronald Macatangay, Charun Upara, Yanee Tanjai, Pornchai Supnithi, Jirapoom Budtho and Pakkapawn Prapan for helping to study the water vapor correction.

\section{References}

[1] Bieber, J. W., Clem, J. M., Duding, M. L., et al. 2004, ApJ, 601, L103

[2] Ruffolo, D., Sáiz, A., Mangeard, P.-S., et al. 2016, ApJ, 817, 38

[3] Mangeard, P.-S., Ruffolo, D., Sáiz, A., et al. 2016b, JGRA, 121, 11620

[4] Sáiz, A., Mitthumsiri, W., Ruffolo, D., Evenson, P., \& Nutaro, T. 2017, Proc. 35th ICRC (Busan), 47

[5] Moraal, H., Potgeiter, M. S., Stoker, P. H., \& van der Walt, A. J., 1989, JGR, 94, 1459

[6] Nuntiyakul, W., Evenson, P., Ruffolo, D., et al. 2014, ApJ, 795, 11

[7] Mangeard, P.-S., Clem, J., Evenson, P., et al. 2018, ApJ, 858, 43 


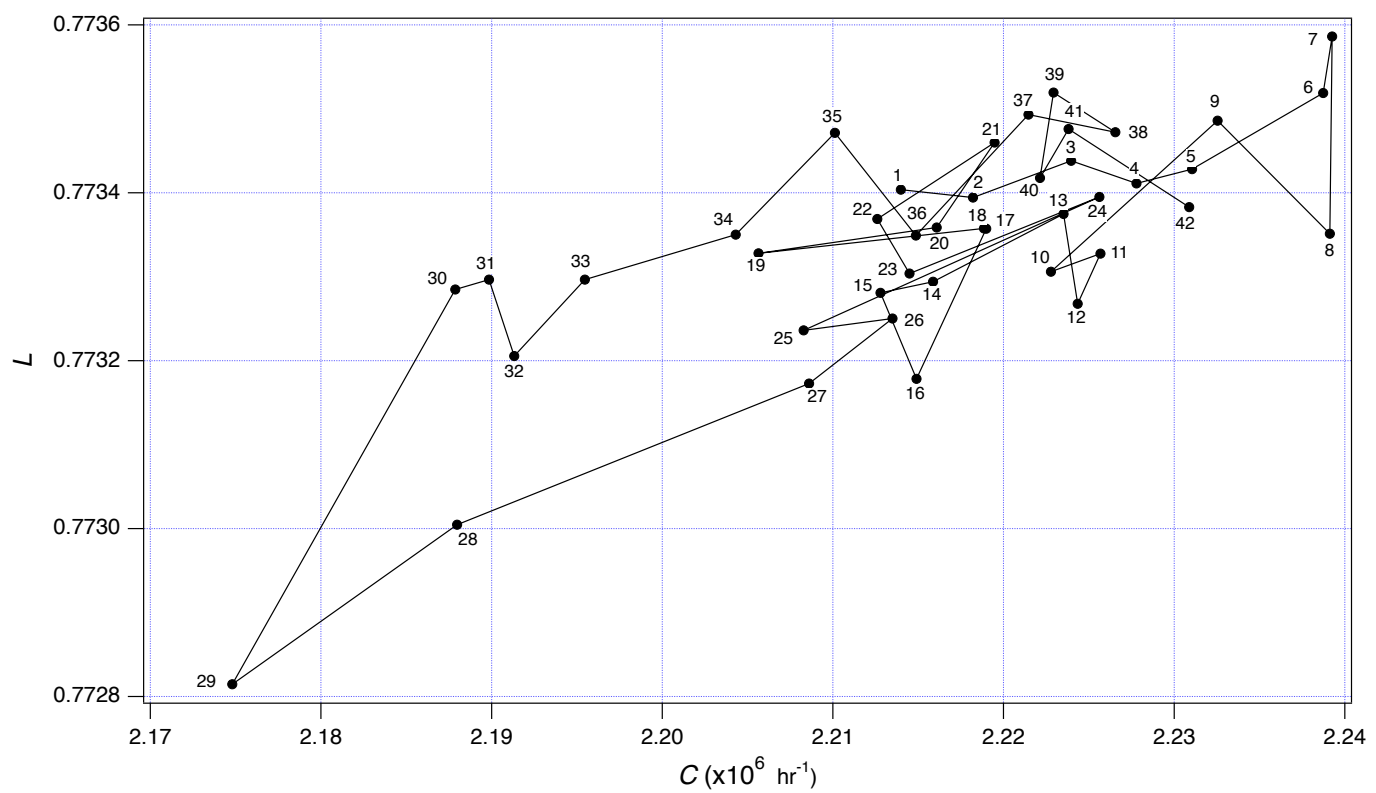

Figure 3: Three-month averages of the leader fraction $L$ vs. count rate $C$ (in counts per hour) in the 18NM64 at Doi Inthanon during 2007 Dec 9 to 2018 Apr 19. The hysteresis indicates that for solar modulation over this period, the spectrum did not "roll" down and "unroll" back up in the same way.

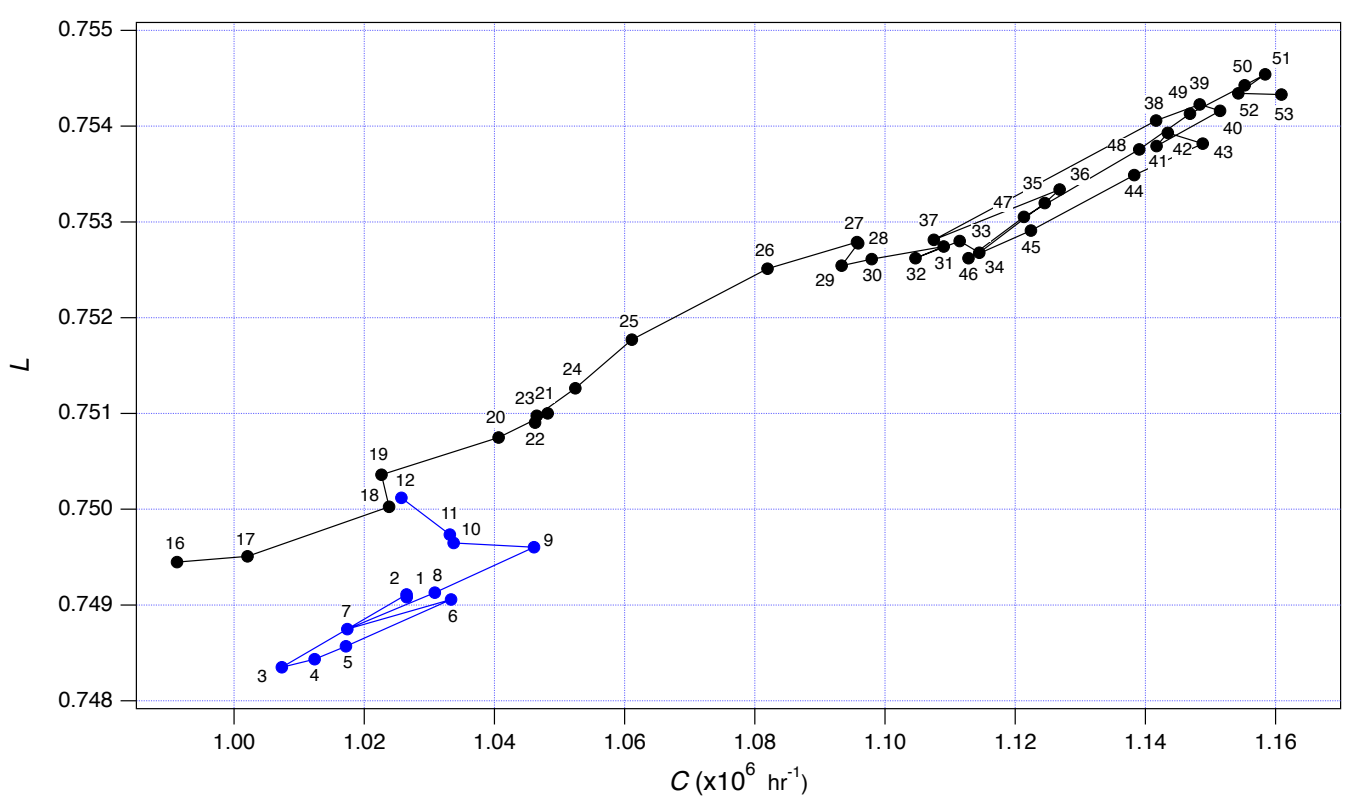

Figure 4: Monthly averages of the leader fraction $L$ vs. count rate $C$ (in counts per hour) in the $3 \times 1$ NM64 at South Pole from 2013 Dec (labelled "1") through 2018 Apr (labelled "53"). Blue coloring indicates that the relative normalization is uncertain. 